\title{
Principes structurants et construction de savoirs en sciences de la vie et de la Terre
}

Denise Orange Ravachol et Françoise Beorchia: Structuring principles and construction of scientific knowledge in life an Earth sciences

\section{Denise Orange Ravachol et Françoise Beorchia}

\section{(2) OpenEdition}

Journals

Édition électronique

URL : https://journals.openedition.org/educationdidactique/1016

DOI : 10.4000/educationdidactique.1016

ISSN : 2111-4838

\section{Éditeur}

Presses universitaires de Rennes

Édition imprimée

Date de publication : 30 mai 2011

Pagination : 7-28

ISBN : 978-2-7535-1449-2

ISSN : 1956-3485

\section{Référence électronique}

Denise Orange Ravachol et Françoise Beorchia, «Principes structurants et construction de savoirs en sciences de la vie et de la Terre », Éducation et didactique [En ligne], 5-1 | 2011, mis en ligne le 30 mai 2013, consulté le 25 avril 2022. URL : http://journals.openedition.org/educationdidactique/1016 ; DOI https://doi.org/10.4000/educationdidactique.1016

\section{Ce document a été généré automatiquement le 25 avril 2022}

Tous droits réservés 


\title{
Principes structurants et construction de savoirs en sciences de la vie et de la Terre
}

\author{
Denise Orange Ravachol et Françoise Beorchia: Structuring principles and \\ construction of scientific knowledge in life an Earth sciences
}

Denise Orange Ravachol et Françoise Beorchia

\section{Introduction}

Dans une étude récente (Orange Ravachol \& Beorchia, 2007), nous avons engagé une réflexion sur l'importance de principes structurants dans la construction des problèmes et des savoirs en sciences de la vie et de la Terre. Ces principes, qui ont historiquement fondé des domaines de savoirs scientifiques, se sont construits contre des idées de la pensée commune. Or il apparaît qu'un certain nombre de difficultés des élèves viennent $d u$ fait qu'ils ne s'emparent pas vraiment de ces principes qui sont pourtant au cœur même du travail des scientifiques. De plus, dans l'enseignement actuel, leur mobilisation semble plutôt aller de soi : même s'ils sont sous-jacents, leur affichage dans les programmes est limité et les pratiques d'enseignement ne contribuent pas vraiment à leur mobilisation, alors que leur rôle est reconnu dans la communauté des chercheurs. En nous appuyant sur nos travaux respectifs en didactique et en épistémologie, nous voulons questionner plus encore leur fonction structurante. Notre choix porte sur le principe de l'actualisme, le principe de l'unité de fonctionnement des animaux et des végétaux et le principe de l'unité de fonctionnement des cellules nerveuses.

2 Notre étude est conduite dans un cadre où la construction d'un savoir scientifique relève d'une problématisation. L'activité scientifique valorise le travail des problèmes explicatifs et elle produit des textes de savoirs articulant étroitement les solutions des problèmes aux raisons qui font qu'elles sont retenues. Dans une première partie, nous présentons ce positionnement théorique. Dans les trois parties suivantes, nous 
précisons les fonctions des principes de l'actualisme, de l'unité de fonctionnement des animaux et des végétaux et de l'unité de fonctionnement des cellules nerveuses dans la problématisation des chercheurs en géologie et en biologie. Nous convoquons notamment les travaux du géologue Lyell (1797-1875), du médecin et physiologiste Claude Bernard (1813-1878) et du neurophysiologiste Sherrington (1857-1952). Fortes de ces approfondissements épistémologiques, nous portons attention dans une nouvelle partie aux rapports qu'entretiennent les élèves du collège, du lycée et du supérieur aux objets de ces sciences et à leurs relations avec ces principes structurants. Nous dégageons enfin des pistes à investir pour la classe et pour la recherche.

\section{Problématisation et construction de savoir scientifique apodictique}

3 Les sciences de la vie et de la Terre se préoccupent tout à la fois d'expliquer le fonctionnement et de reconstituer l'histoire des systèmes complexes que sont notamment l'organisme vivant ou la planète Terre. Comme toute science, elles accordent une large place à l'imagination, qu'elles mettent sous le contrôle de l'observation, de l'expérience et de la critique, car il s'agit à chaque étape de leur démarche de «limiter la part du rêve dans l'image du monde » qu'elles élaborent (Jacob, 1981 ; Popper, 1972/1985). Les textes de savoir qu'elles produisent peuvent ainsi se démarquer des mythes.

\section{L'activité scientifique comme la construction d'un troisième monde}

4 Sans admettre un exact parallélisme entre le fonctionnement de la communauté scientifique de référence et celui de la classe, du fait notamment des différences de temporalité où ils s'exercent, la connaissance du premier peut cependant aider à comprendre le second, en donnant à voir des aspects qui ne seraient pas forcément pris en compte dans les situations scolaires. Ainsi en est-il des caractéristiques des savoirs qui se construisent et des textes qui les supportent. Ces savoirs gagnent en généralité (ils dépassent le ou les cas ayant servi à les élaborer), en universalité (ils prennent du sens au-delà de la communauté qui les a construits) et en apodicticité (c'est-à-dire qu'ils ne se limitent pas aux solutions du problème mais intègrent également ce qui fait que ce sont ces solutions et pas d'autres qui sont retenues). Encore faut-il bien préciser ce qu'ils représentent. Pour suivre Popper (1979/1991), nous dirons que les savoirs scientifiques se dégagent d'un monde de sujets et qu'ils participent d'un "troisième monde », objectif et autonome, dont les « habitants » sont les systèmes théoriques, les problèmes et états de ces problèmes (état des discussions, état des échanges d'arguments critiques). Notons que paradoxalement, ce monde «sans sujet connaissant " ne peut être compris que des sujets capables de le réinterroger jusque dans les pratiques parties prenantes de sa production. De plus, qui dit état des problèmes, dit inscription de ces problèmes dans le fonctionnement à un moment donné de la communauté scientifique, mais aussi enracinement de ces problèmes dans une histoire de la connaissance. Pour suivre Bachelard, ce n'est pas une évolution continuiste du discours scientifique qu'il faut convoquer, comme si «la science d'aujourd'hui est toujours à quelque degré annoncée dans le passé » (Canguilhem, 1983, p.178). Il s'agit de prendre en compte "l'histoire des défaites de l'irrationalisme » 
(ibidem, p. 183) et de remonter de l'actuel vers les commencements «en sorte qu'une partie seulement de ce qui se donnait hier pour science se trouve à quelque degré fondée par le présent » (ibid., p. 178).

\section{L'activité scientifique comme un processus de problématisation}

5 Nous concevons l'activité scientifique comme la prise en charge et le travail de problèmes explicatifs aboutissant à la production de savoirs scientifiques raisonnés, ce que Fabre, Orange et leur équipe nomment une problématisation (voir notamment Fabre \& Orange, 1997). Il faut voir la problématisation comme un processus d'ouverture à des explications possibles (exploration d'un espace de contraintes) et de fermeture (construction de nécessités) permettant de construire des savoirs apodictiques. Dans une telle démarche, ce qui est difficile est la construction des raisons contraignant les solutions et la mise en texte de cet ensemble. La première repose sur l'élaboration/ articulation de modèles explicatifs et de données empiriques (observation, expérimentation, documentation) ; la seconde exige de penser l'incorporation au texte d'éléments de pratiques. Le savoir scientifique tient ainsi sur la délimitation et la mise en fonctionnement d'un espace du problème, où les pratiques langagières, et notamment les débats, jouent un rôle important. Une telle approche conduit à penser une forme renouvelée du texte du savoir. Il ne peut se suffire des solutions, cela tirerait vers la pensée commune. Il doit rendre compte des pratiques qui les sous-tendent. C'est pourquoi nous portons attention au travail des idées des chercheurs et des élèves dans les moments d'échanges, de débat, de controverse, parce qu'ils sont des lieux propices aux développements d'arguments et à la construction de raisons, par une exploration du possible articulée à une extraction du nécessaire, empêchant ainsi « d'emprunter un circuit trop court des idées aux raisons » (Bachelard, 1938, p. 44).

Ces repères installent le cadre théorique de notre réflexion sur les principes structurants en sciences de la vie et de la Terre. Nous allons les mobiliser dans l'étude successive du principe de l'actualisme, du principe d'unité de fonctionnement des animaux et des végétaux et du principe d'unité de fonctionnement des cellules nerveuses.

\section{Le principe de l'actualisme, principe structurant de la géologie historique}

7 La géologie tente d'expliquer le fonctionnement actuel de la Terre et d'établir son histoire, une histoire révolue donc unique, dont la reconstitution est difficile, notamment parce qu'elle explore des périodes qui vont bien au-delà de la présence humaine, qu'elle ouvre donc sur une diversité de scénarios possibles, et qu'il se pose le problème de leur contrôle.

\section{Les spécificités de la géologie historique}

8 Comment construire de manière raisonnée des phénomènes et des événements anciens qui ont marqué la Terre et qui peuvent rendre compte de son état actuel ? Gould (1989, p. 282) spécifie les démarches : 
"We search for repeated pattern, shown by evidence so abundant and so diverse that no other coordinating interpretation could stand, even though any item, taken separately, would not provide conclusive proof.»

Les géologues rangent parmi ces données ("evidence») les archives de la Terre, vues comme des traces de phénomènes ou d'événements. Mais elles ne relèvent pas d'un empirisme immédiat. Si elles paraissent dignes d'intérêt, c'est qu'elles représentent des saillances spatiales et/ou temporelles faisant écho au questionnement des chercheurs et à leur expérience humaine du monde. Les édifices volcaniques du Massif Central, par exemple, rappellent des édifices volcaniques actuels et interrogent sur le volcanisme. C'est par la mise en comparaison répétée d'éléments du passé avec des phénomènes actuels qu'il est possible de reconstruire, au moins par morceaux, une histoire de la Terre. Mais cette histoire ne suffit pas : il est en effet difficile de penser que la Terre actuelle exhibe tous les processus et toutes les traces de son passé. Le jeu entre le passé et l'actuel est donc nécessaire mais il montre des limites.

11 La marque de l'histoire n'est pas seulement celle des phénomènes encore à l'œuvre de nos jours. Elle peut être celle d'événements, par définition singuliers dans le temps et l'espace, et dont la survenue tient de la contingence: ils auraient pu ne pas être. Comment expliquer leur survenue? En fait, ils sont identifiés lorsqu'ils deviennent nécessaires. Il s'agit alors de rechercher leurs conditions de possibilité que seule une logique rétrospective permet d'envisager (ibidem, p. 283). En effet, procéder dans une expérience de pensée à un "rembobinage du film » puis penser l'histoire dans le sens de son flot expose, à cause de la contingence, à une infinité de parcours possibles : à la place de chaque événement construit, d'autres auraient pu se produire. La construction des événements se fait donc selon une logique à contre-courant de l'histoire. L'événement E (par exemple l'éruption de la montagne Pelée, dans l'île de la Martinique, en 1902) est contingent en ce sens qu'il dépend d'une séquence non déterminée d'états antécédents. Il est d'autant plus réel (en anglais « actual ») qu'il est davantage contraint par des traces observables.

\section{Le principe méthodologique de l'actualisme}

12 Le principe méthodologique de l'actualisme (on dit aussi principe des causes actuelles') tient une grande importance dans le développement de la géologie comme une science historique. Comme l'écrit Hooykaas (1963, p. XII), ce principe est :

13 " the bridge that enables our imagination to go from the present to the past, to visualize with some confidence that no human eye has seen ".

14 En quoi ce principe consiste-t-il ? Il affirme que « le présent est la clé du passé, où que les causes qui ont agi au long de l'histoire de la Terre ne diffèrent point essentiellement des causes géologiques actuelles ${ }^{2}$ (érosion, transport, sédimentation, métamorphisme, volcanisme, plissement et soulèvement des montagnes) »(Gohau, 1997, p. 140). Et, pour reprendre encore cet auteur, l'actualisme en géologie est « la transposition au temps du postulat d'universalité des lois de la nature, postulat qu'on trouve chez Galilée quand il montre que le monde supralunaire d'Aristote est de même nature que le monde infralunaire, et que les étoiles ne sont pas moins corruptibles que les objets d'ici-bas, comme le prouve l'apparition des novae » (Gohau, 2003, p. 10).

Nous avons donc affaire à une sorte de postulat dont la force tient au fait qu'il permet de contrôler les reconstitutions possibles de l'histoire de la Terre. Dans l'espace des 
contraintes représentant le processus de problématisation, nous le situons dans le registre explicatif, «le monde qui donne sens au modèle et permet de le manipuler » (Orange, 1997).

\section{La complexité du principe de l'actualisme}

16 En nous référant à la naissance de la géologie, de la fin du XVII e siècle au début du XIX ${ }^{e}$ siècle, nous pouvons entrer dans la complexité du principe de l'actualisme. Ce fut le moment où les géologues ont pris des distances avec les cosmogonies et les géogonies ${ }^{3}$, trop spéculatives à leur goût pour être scientifiques, et où les explications strictement déterministes comme celles de Descartes (1596-1650) leur ont paru insuffisantes. Ils ont voulu se limiter à l'explication des changements géologiques dont les marques étaient encore accessibles à l'observation, et ils « ont tenté de le faire en recourant aux causes qu'ils voyaient agir sous leurs yeux» (Hooykaas, 1970, p. 19). Depuis, leur démarche contient toujours une part d'inférence à partir du présent, de ce qui, des processus à l'œuvre aujourd'hui, leur est connu et accessible. On saisit alors l'intérêt de se doter "d'une vaste batterie, (d') un riche assortiment de " causes actuelles » connues à fond, (d'un) un riche trousseau à clefs à essayer dans les vieilles serrures, sans forcer, prudemment » (Ellenberger, 1996, p. 66). Mais tous les géologues ne reconstruisent pas la même histoire de la Terre. De là des controverses. Celles du XIXe siècle opposent deux courants de pensée : l'uniformitarisme (un actualisme strict) et le catastrophisme.

17 Le courant uniformitarien, ou actualiste s'en tient à des causes encore agissantes. Le courant catastrophiste ${ }^{4}$ met en jeu des causes de nature et/ou d'intensité différente des causes encore agissantes. Cette tension entre deux pôles aide à cerner la complexité du principe de l'actualisme. En effet, tous les géologues, même les catastrophistes, admettent que le présent est la clé du passé. «À moins de décréter que l'on va purement et simplement inventer a priori l'histoire passée de la Terre, le plus simple bon sens impose de partir des données concrètes offertes par la géographie et par le contenu du sous-sol » écrit F. Ellenberger (1994, p. 12). Le principe de l'actualisme s'impose donc contre les reconstitutions fantastiques du passé de la Terre, c'est-à-dire contre un catastrophisme immédiat et de circonstance. Tous les géologues ne font pas cependant le même pari sur le passé, autrement dit ne construisent pas le même monde de possibles, et ils ne font pas exactement le même recours au présent, ils ne construisent pas les problèmes de la même manière.

18 - Certains géologues (Hutton 1726-1797 ; Lyell, 1797-1875) conçoivent la Terre dans un état d'uniformité du monde physique et du monde vivant. Certes, des changements perturbent cet état en chaque lieu mais ils sont compensés par d'autres se produisant ailleurs ou un peu plus tard. Avec un tel pari, qu'en est-il du problème de la formation des montagnes? Puisque la nature actuelle ne donne pas à voir de telles surrections, le principe de l'actualisme oblige à construire la nécessité des longues durées constructrices $\mathrm{du}$ phénomène d'orogenèse (un actualisme de $2^{\mathrm{e}}$ niveau, Orange Ravachol, 2003a, 2005). L'actualisme se pose donc contre un catastrophisme immédiat mais il tend à enfermer la Terre dans du temps profond (" No vestige of a beginning, no prospect for an end » écrit Hutton) et, de ce fait, il évacue une histoire globale de la Terre.

19 -- D'autres géologues (de Beaumont, 1798-1874; les géologues actuels) s'inscrivent dans un schéma directionnaliste où la Terre a une histoire sagittale irréversible. Dans un tel pari, le principe de l'actualisme dans sa version stricte est mis à mal puisque les 
conservations de la nature et de l'intensité des causes géologiques sont difficilement tenables. Ce principe se heurte donc au directionnalisme, qui conduit à penser que la nature actuelle ne contient pas toutes les causes géologiques ayant existé et à envisager des causes anciennes d'une autre nature, sans qu'elles soient forcément d'une autre intensité que les causes actuelles. L'explication de la formation d'une chaîne de montagnes que construit Elie de Beaumont l'illustre. En mettant en jeu des soulèvements de couches provoqués par la contraction d'une Terre se refroidissant progressivement, soit une cause toujours existante et d'intensité constante ${ }^{5}$, elle use du principe de l'actualisme. En convoquant les phases tectoniques d'orogenèse et de déformations, qui génèrent quasi instantanément des entités tectoniques régulières et statiques et que le présent n'offre pas, elle s'en écarte ${ }^{6}$. Mais cette convocation de catastrophes ne relève pas d'une solution de facilité ; elle vient quand l'actualisme est porté à ses limites.

20 Avec le $\mathrm{xx}^{\mathrm{e}}$ siècle, "la longue domination de l'actualisme " s'installe (Babin, 2005, p. 132). Il est pourtant des prises de position ou des travaux qui le remettent sur le devant de la scène. En voici quelques exemples? .

21 - C'est pour partie en référence à l'actualisme que le paléontologue américain Schuchert rejette le mobilisme wégenérien (Oreskes, 2001). Pour ce partisan des ponts continentaux, « the stopping point lay not in physics but in the practice of geological geology. A few hundred miles could be tolerated, because that would leave faunal zones intact. But a few thousand miles would mean a radical rethinking of his uniformitarianism " (Oreskes, 1999, p. 202). Et d'affirmer en 1928 que "we are on safe grounds only so long as we follow the teachings of the law of uniformity in the operation of nature's laws " (ibidem, p. 202).

- Dans l'article intitulé Is uniformitarianism necessary? publié en 1965 et depuis fréquemment cité dès lors qu'il s'agit d'actualisme, Gould écrit que : « As a special term, methodological uniformitarianism was useful only when science was debating the status of the supernatural in its realm; for if God intervenes, then laws are not invariant and induction becomes invalid» (ibidem, p. 223). C'est dire que, la bataille contre les explications surnaturelles ou divines étant désormais gagnée ${ }^{8}$, «the term today is an anachronism» (ibidem, p. 227). Cet article suscite pourtant des réactions vigoureuses, allant jusqu'à obliger Gould à une mise au point où il dit son attachement au principe «I did not suggest that we abandon the present in our search to understand the operation of past processes " (Gould, 1965b, p. 919).

23 Le principe méthodologique de l'actualisme est donc nécessaire pour penser scientifiquement les problèmes de la géologie historique : il oblige à les construire quand un catastrophisme immédiat les évacue. Mais c'est un postulat d'une utilisation exigeante. Il confère une complexité certaine au présent, comme produit de l'histoire (le présent porte des traces du passé qu'il s'agit d'identifier et d'expliquer) et vivier de modélisation du passé de la Terre permettant d'expliquer les traces (le présent est le siège de processus en action). Il ouvre de plus sur des tensions: en permettant d'exporter des processus actuels dans le passé, il contre la possibilité d'une histoire directionnaliste et événementielle mais parce que la nature actuelle ne peut contenir tous les processus il permet de construire des nécessités de l'histoire (nécessités du temps long producteur de phénomènes, nécessité d'événements historiques). Et, si l'invariabilité des lois physiques est incontestée, des discussions peuvent toujours exister du fait que ce principe mobilise des causes géologiques complexes. 


\section{Le principe d'unité de fonctionnement des animaux et des végétaux, principe structurant de la physiologie}

\section{Les tensions des sciences physiologiques}

Les sciences physiologiques naissent véritablement au milieu du XIX siècle. C'est le moment où «aussi bien par la voie de l'histoire naturelle que par celle de la physiologie, l'organisme vivant pouvait être considéré comme un objet dont le mode d'existence est susceptible d'une valorisation contrastée » (Canguilhem, 1988, p. 132). C'est aussi à ce moment que la tension de la physiologie entre science historique et science fonctionnaliste (Mayr, 1989) commence à véritablement s'exprimer. En effet, les problèmes qui ont de l'importance concernent non seulement le fonctionnement des organismes mais aussi leur origine. La physiologie naissante est marquée d'un courant vitaliste (dans le sillage des travaux de Bichat, 1771-1802) où des forces vitales étrangères aux lois physiques sont prégnantes et d'un courant mécaniste tendant à s'en remettre aux seules lois physico-chimiques (Magendie, 1783-1855). Et, avec la diffusion des travaux de Darwin, un courant évolutionniste se pose contre les différentes théologies en s'emparant de la question de l'origine des espèces.

\section{Le principe d'unité de fonctionnement des animaux et des végétaux}

Les travaux de Claude Bernard (1813-1878) installent les conditions d'émergence de la physiologie en rassemblant dans une même unité de fonctionnement les animaux et les végétaux. Jusqu'alors les scientifiques subdivisent le monde vivant en deux règnes, le règne animal et le règne végétal, tenus par des relations de complémentarité: les végétaux élaborent le sucre et plus largement la matière organique dont les animaux se nourrissent à des fins énergétiques et plastiques. Sur la base de nombreuses expériences (Grmeck, 1997) Bernard établit que des organismes animaux exclusivement nourris de viande ont un sang contenant du sucre, donc qu'ils produisent du sucre. Il réfère cette production au fonctionnement $\mathrm{du}$ foie, capable de fabriquer et de stocker du sucre sous forme de glycogène (glycogénogenèse) et d'en libérer à partir de ce glycogène (glycogénolyse). Le modèle de nutrition directe, pour qui la matière végétale prélevée par les animaux sert à faire de la matière animale, est remis en cause au profit d'un modèle de nutrition indirecte: les animaux stockent les molécules (dont les sucres) en provenance de l'alimentation puis utilisent ces réserves après qu'ils les ont éventuellement transformées. Cela a deux conséquences: 1) L'organisme animal conquiert une autonomie (liée au milieu intérieur, nous y reviendrons plus loin) dont le privait la seule nutrition directe ; 2) Le règne animal ne peut plus être séparé du règne végétal. Comme l'extrait suivant l'atteste, toute la physiologie de Bernard dérive de son dépassement de l'opposition entre les animaux et les végétaux :

« il n'en reste pas moins démontré ce fait capital qu'il existe une fonction glycogénique chez les animaux comme chez les végétaux. Nous savons que cette fonction glycogénique est liée intimement dans les deux règnes aux phénomènes de développement chez l'embryon et aux phénomènes de nutrition chez l'adulte, phénomènes qui sont de même nature. De sorte que les points obscurs, qui restent encore à éclaircir sont relatifs à la physiologie générale qui embrasse tous les êtres vivants dans son domaine » (Bernard, 1877, p. 547). 


\section{Le dépassement de l'opposition vitalisme-mécanisme} s'appuyant sur la théorie darwinienne de l'évolution, Sherrington (1857-1952) engage la construction du concept d'intégration nerveuse à la fin du XIXe. Il va le développer dans son ouvrage principal, The integrative Action of the Nervous System (1906), en étudiant principalement les réflexes spinaux des mammifères, mais en faisant référence aussi à d'autres espèces animales. La construction du concept d'intégration est donc soustendue par le principe d'unité de fonctionnement des cellules nerveuses, qui va aussi conditionner l'émergence de concepts associés (synapse, inhibition...). 


\section{Construction du concept d'intégration et des concepts associés}

31 Sherrington situe ses recherches dans le cadre de la théorie cellulaire (Schwann, 1839; Deiters, 1865, pour son application aux cellules nerveuses) et dans celui de la théorie darwinienne de l'évolution (Darwin, 1859) :

- La cellule est l'unité de structure du vivant. Reprenant le concept de milieu intérieur de Bernard (voir la section 3 de cet article), Sherrington va établir le parallèle avec le système nerveux pour expliquer comment un organisme qui atteint une certaine dimension, donc un grand nombre de cellules et d'organes distincts, peut s'adapter aux stimuli issus de son environnement.

- La théorie darwinienne de l'évolution établit à la fois des filiations entre les espèces et les groupes animaux et donc explique les ressemblances dans leur organisation. Sherrington met en avant dans tout son ouvrage des comparaisons entre les systèmes nerveux des organismes, des éléments nerveux diffus de certains (méduses, etc.) aux différents niveaux d'organisation du système nerveux chez d'autres (arthropodes, vertébrés).

Ne se limitant pas à considérer l'unité de structures, Sherrington adopte le principe d'unité de fonctionnement des cellules nerveuses tout en constatant certaines différences d'organisation.

Dans un contexte de controverses entre réticularistes et neuronistes, Sherrington se place du côté des neuronistes: en s'appuyant sur les méthodes de coloration développées par Golgi, il prend position pour la contiguïté, et non la continuité, entre deux neurones. Mais il conçoit la synapse non comme une structure identifiable (ce qui n'est pas possible à ce moment étant donné les techniques de microscopie) mais inférable grâce aux travaux récents de Ramon Y Cajal: c'est sans aucun doute un élément indispensable dans sa théorie de la fonction intégrative du système nerveux. Il va lui donner le nom de synapse (avec Foster en 1897).

Les expérimentations menées par Sherrington portent sur des réflexes chez des animaux spinaux : il compare les caractéristiques des conductions et réponses dans ces réflexes à celles obtenues dans les troncs nerveux et constate de nombreuses différences. Certains faits expérimentaux s'imposent comme contraintes empiriques (des faits qu'il s'agit d'expliquer et dont il faut tenir compte pour expliquer) car ils ne correspondent pas aux résultats habituellement obtenus dans les troncs nerveux (c'està-dire les éléments nerveux extérieurs aux centres nerveux et les structures ganglionnaires). Il attribue ces différences aux constituants de la substance grise, mais il élimine progressivement certains éléments pour tenir compte de résultats expérimentaux et d'observations faites par différents scientifiques. Il est ainsi conduit à conclure à l'existence de surfaces de séparation entre neurones, situées dans la substance grise.

37 S'appuyant donc sur le principe d'unité de structure et de fonctionnement des cellules nerveuses, Sherrington $(1897,1906)$ construit le concept de synapse et s'en sert pour interpréter les résultats expérimentaux sur les réflexes spinaux. Cela lui permet aussi de concevoir une modélisation de l'intégration qui est valide par rapport aux résultats obtenus. Ce qui dans la synapse est important est l'existence d'une surface de discontinuité, une barrière membranaire qui permet d'expliquer les changements physico-chimiques qui peuvent être responsables à la fois des processus d'excitation 
mais aussi d'inhibition. Enfin la présence des synapses est généralisable aux différents centres nerveux.

\section{Dépassement d'une conception centraliste du fonctionnement cérébral}

38 Le principe de l'unité structurale et fonctionnelle du système nerveux basée sur les concepts de neurone et de synapse s'oppose à une conception centraliste dans laquelle toute l'activité nerveuse est issue du cerveau. Adopter le principe d'unité de fonctionnement des neurones dans les différentes parties du système ne veut pas dire renoncer à un fonctionnement coordonné et contrôlé au contraire : c'est accepter que cette coordination se fasse à différents niveaux. Pour reprendre Canguilhem (1955, p. 171), c'est un système de systèmes, ou système de systématisation :

39 "On ne pouvait s'attendre à voir s'effondrer du premier coup la notion très antique d'un centre, hiérarchiquement premier, de la régulation des mouvements de l'organisme. Avant de concevoir le système nerveux, avec Sherrington, comme un système de systématisation, il fallait d'abord le concevoir, avec Unzer et Prochaska, comme un système de systèmes. »

Le choix de ce principe est fondateur dans la mesure où il contribue à asseoir les bases de la neurophysiologie, et heuristique en particulier car il fournit un modèle (celui de la synapse) susceptible d'être généralisé à l'ensemble des structures nerveuses.

\section{Des principes des chercheurs au fonctionnement des élèves}

41 Des approfondissements épistémologiques précédents, nous dégageons les rôles structurants et heuristiques du principe de l'actualisme, du principe d'unité de fonctionnement des animaux et des végétaux, du principe d'unité de fonctionnement des cellules nerveuses, et l'importance qu'ils prennent dans un contexte de controverses (uniformitarisme/directionnalisme, vitalisme/mécanisme ; réticularisme/ neuronisme). En nous appuyant sur ces repères, voyons de plus près comment les élèves s'approprient les problèmes de géologie historique, de physiologie et de neurophysiologie et le rapport qu'entretient leur raisonnement à ces principes.

\section{Des collégiens et des lycéens et la mise en jeu du principe de l'actualisme}

42 C'est au travers de leur confrontation au problème de la formation d'une chaîne de montagnes (chaîne alpine, Himalaya), et avant enseignement, que nous allons étudier comment des collégiens et des lycéens ${ }^{9}$ mobilisent le principe méthodologique de l'actualisme.

\section{La mobilisation d'une forme élaborée du principe de l'actualisme}

Conformément à ce que font les géologues contemporains, le travail de ce problème en classe de sciences consiste à reconstituer un scénario vraisemblable d'orogenèse par 
une mise en jeu contrôlée de plaques tectoniques. La nature actuelle ne donnant pas à voir de formation de chaînes de montagnes, il est nécessaire d'envisager un processus actuel producteur de relief et de structures et de l'imaginer sur une durée qui dépasse le temps humain, soit une forme élaborée du principe de l'actualisme (Orange Ravachol, 2003a, 2005).

Le travail de ce problème repose aussi sur la mobilisation de traces dont la recherche n'a rien d'aisé. En effet, elles ne peuvent pas être en grand nombre, soit qu'elles ont été détruites, soit qu'il est difficile de les identifier (Celâl Sengör, 2005). Parmi celles-ci, des pillow lavas, semblables aux roches formées actuellement au niveau des dorsales océaniques, et la présence de strates sédimentaires plissées, qui obligent à envisager des phénomènes d'accrétion océanique (donc un contexte de divergence de plaques) et des phénomènes de compression (donc un contexte de convergence de plaques), et en conséquence un passage du premier régime au second. Parce que les pillow lavas se trouvent maintenant en altitude, il est nécessaire de convoquer une surrection des formations rocheuses et un temps long constructeur de ce phénomène. La figure 1 donne à voir l'espace des contraintes pouvant être développé. La construction de l'histoire "réelle" d'une chaîne de montagnes combine donc la convocation de processus se déployant à différentes échelles et elle exige une mise en jeu subtile du temps.

Figure 1. Espace des contraintes envisageables pour le niveau quatrième (grade 8, 13-14 ans)

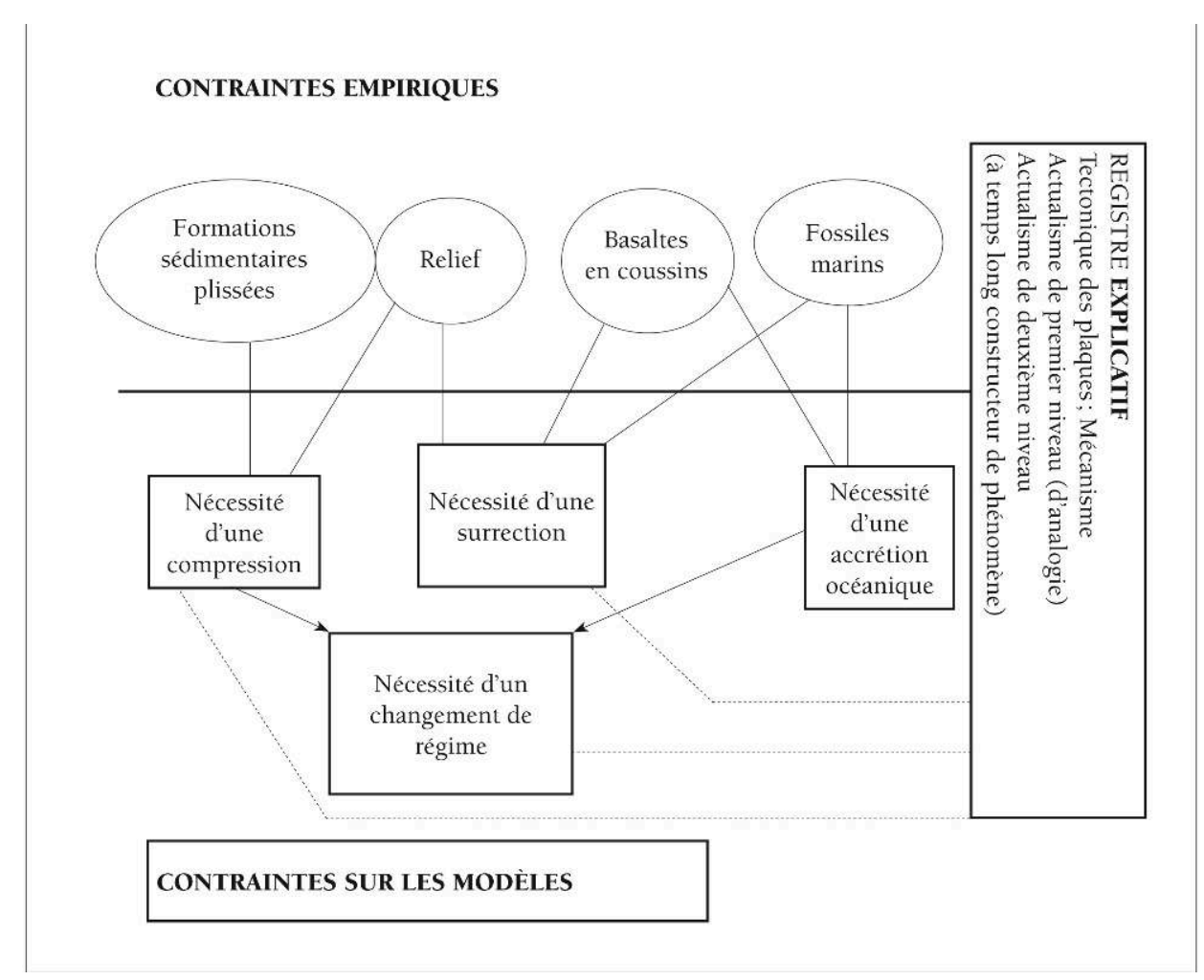

Les caractéristiques du recueil de données

L'expérimentation sur laquelle nous nous basons se situe au début de la séquence d'apprentissage. Elle concerne une classe de 22 élèves de quatrième (grade 8) et une classe de 30 élèves de première scientifique (grade 11): 

22 jeunes élèves (13-14 ans) proposent une réponse mettant en jeu la rencontre et l'affrontement de plaques tectoniques. Ils se cantonnent à l'explication des reliefs, qui font figures de traces à expliquer, en mobilisant un cadre de dynamique de plaques peu contraignant, sans faire appel à des traces, sans poser de contraintes temporelles. Ils sont dans le registre d'une mise en histoire (storytelling, figure 2).

Figure 2. Réponse d'un groupe de 3 collégiens (classe de quatrième, grade 8, 13-14 ans)

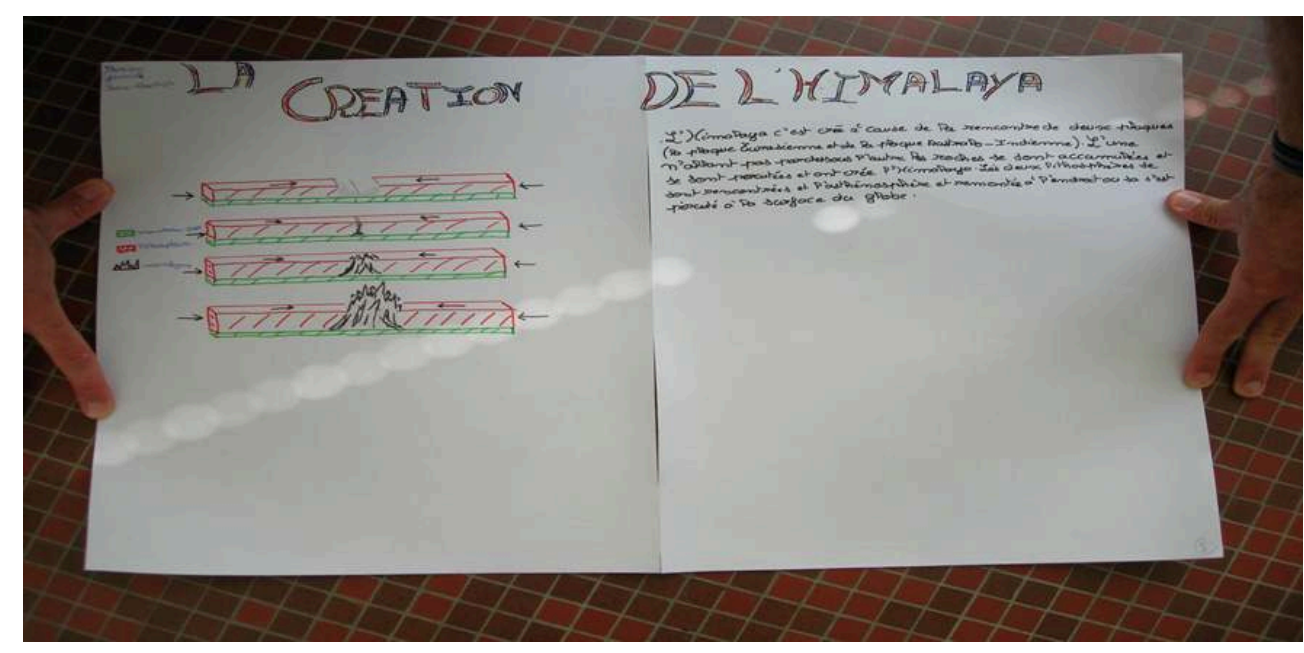

Voyons maintenant ce que proposent des élèves de lycée ayant une certaine connaissance des phénomènes géologiques (en matière de possibles tectoniques et orogéniques notamment). Nous constatons que ces élèves proposent des solutions qui se rapprochent davantage, en apparence, de celles des chercheurs. Mais tout se passe comme s'ils se permettaient tout, sans règle ni contrôle, jusqu'à obtenir la configuration actuelle des formations. Comme des bricoleurs (Lévi Strauss, 1962), ils inventent le montage de la chaîne de montagnes avec les objets (fonds océaniques, plaques, etc.) et les outils (rapprochement, écartement, affrontement, failles inverses, etc.) dont ils disposent (figure 3). Rien de la nature actuelle ne leur sert véritablement de référence, la nécessité du temps long n'est pas construite, et la contingence historique est loin d'être entrevue. Les élèves proposent seulement des solutions à l'obtention d'une figure géologique. C'est la marque évidente d'un manque de 
problématisation géologique, dont la condition réside dans la mobilisation du principe de l'actualisme. Les lycéens eux aussi se rabattent sur une mise en histoire (du storytelling).

Figure 3. Réponse d'un élève de première scientifique (grade 11, 16-17 ans)

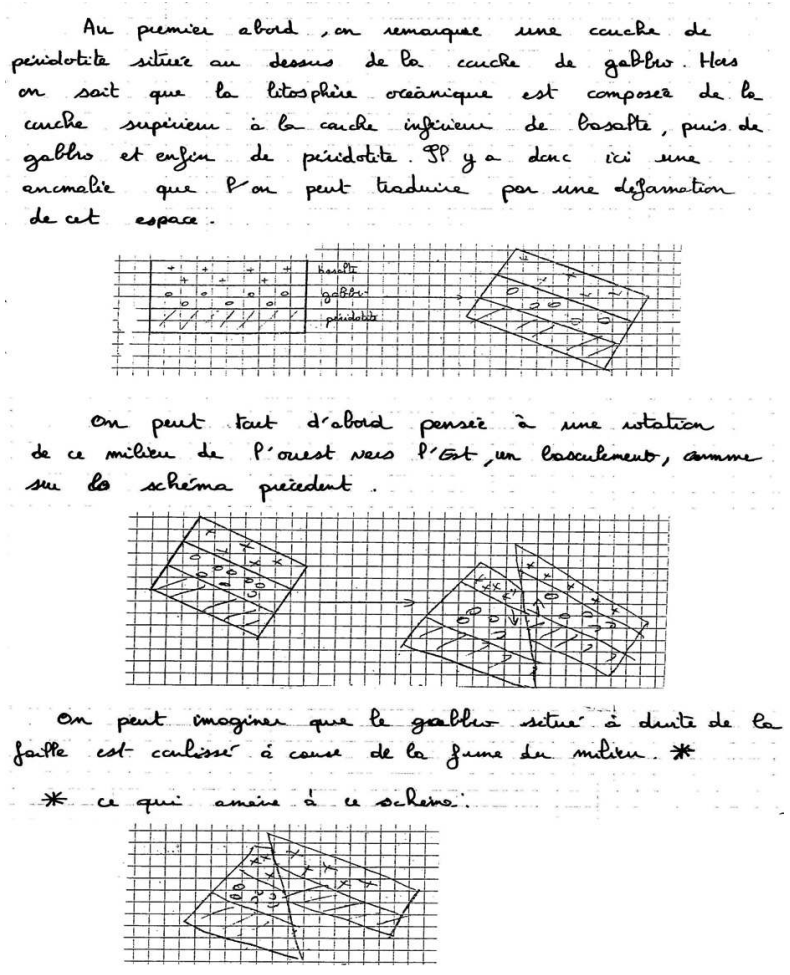

51 Pour ce problème de géologie historique et avant enseignement, les élèves lycéens et collégiens fonctionnent de manière sensiblement différente des chercheurs. Ils mobilisent un actualisme d'analogie et un catastrophisme de $1^{\text {er }}$ niveau (sans prise en compte du temps long). Ils versent davantage vers une mise en histoire (un storytelling) contingente (elle pourrait être autre) et closant le problème.

\section{Des lycéens et la mise en jeu du principe d'unité de fonctionnement des animaux et des végétaux}

52 Nous nous intéressons maintenant aux rapports qu'entretiennent des lycéens (16-17 ans, première $S$, grade 11) avec le principe d'unité des animaux et des végétaux. Nous conduisons cette étude de manière indirecte, en nous focalisant sur la signification que peut prendre chez eux le concept de milieu intérieur. Un premier cas sera consacré au problème de l'approvisionnement des organes en substances nutritives (Orange \& Orange, 1995), le deuxième au renouvellement moléculaire des cellules (Orange Ravachol, 2003b) ${ }^{10}$.

\section{Le problème de l'approvisionnement des organes en substances nutritives}

Dans le cadre de l'étude de quelques aspects de physiologie appliquée à l'activité sportive et après que la classe a clairement identifié les besoins des muscles (approvisionnement en petites molécules organiques et en dioxygène, évacuation des 
déchets produits à savoir $\mathrm{CO} 2$ et déchets azotés), l'enseignant demande aux 34 élèves d'une classe de première scientifique organisés en binômes de représenter par un schéma la circulation du sang de façon à expliquer comment le sang peut approvisionner le muscle en dioxygène.

Les schémas obtenus se classent aisément en 3 catégories selon qu'ils mettent en jeu un simple itinéraire poumon/muscle (type 1, 5 binômes sur 17), une boucle de circulation (type 2, 6 binômes sur 17) ou deux boucles de circulation (type 3, 6 groupes sur 17). Le modèle canonique attendu se confond avec le type $3:$ il assimile la circulation du sang à une double circulation (boucle de circulation pulmonaire et boucle de circulation générale ou systémique). La présentation et la confrontation des différents types d'explication montre une adhésion rapide de tous les élèves au type $3:$ il paraît clair à tous qu'un tel schéma permet d'approvisionner les cellules en oxygène et de récupérer le $\mathrm{CO} 2$.

Le professeur demande alors aux élèves de placer sur ce double circuit, qu'ils peuvent modifier comme ils le veulent, le tube digestif et les reins de façon à satisfaire les autres conditions de la nutrition des cellules (approvisionnement en petites molécules organiques, élimination des déchets azotés). Dans une très grande majorité, ils construisent des modèles du type de celui de la figure $4 \mathrm{a}$ : le tube digestif, les organes (avec les cellules) et les reins y sont disposés en série et dans cet ordre. Il faut comparer cette proposition avec le modèle généralement admis de la circulation sanguine (figure $4 b)$ : organes divers, reins, tube digestif y sont placés en parallèle.

Figure 4. Approvisionnement de l'organisme en substances nutritives et évacuation des déchets 4a. Le modèle d'un groupe d'élèves (Première $S$, grade 11, 16-17 ans)

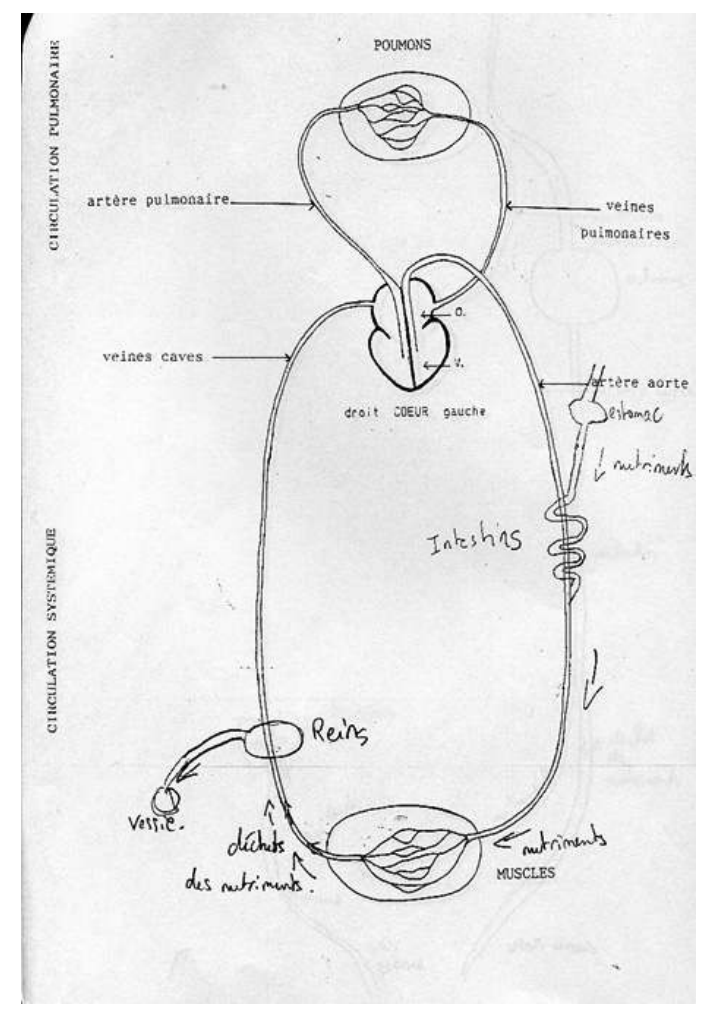




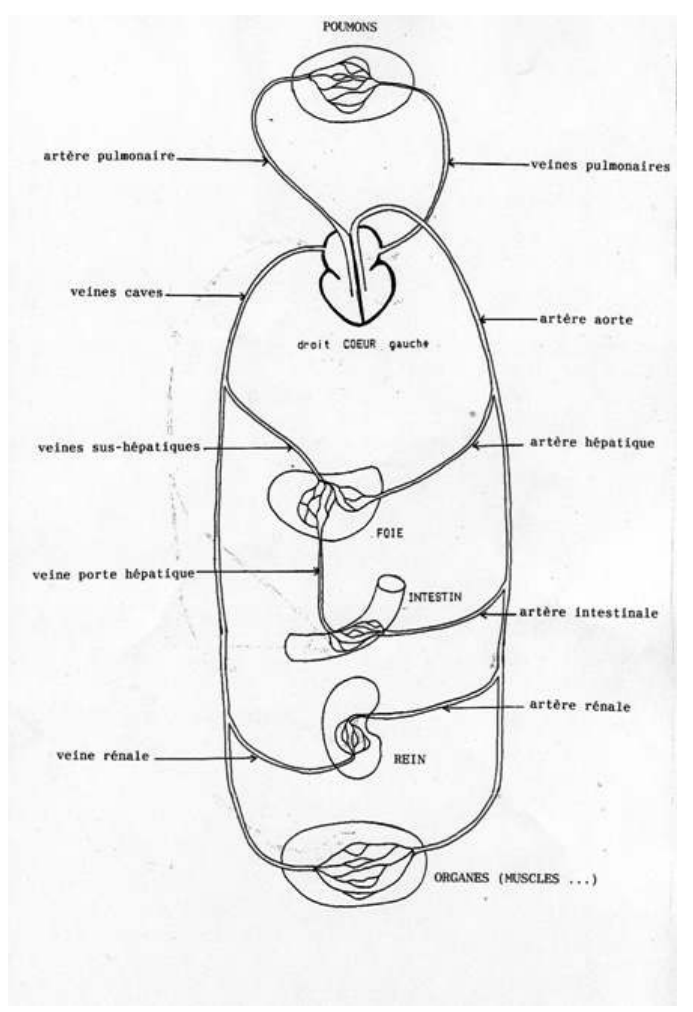

Le raisonnement qui conduit les élèves à une organisation en série est très régulièrement explicité ainsi : le tube digestif doit être placé avant les organes dont on considère la nutrition car il faut que le sang qui leur parvient soit riche en petites molécules organiques. Le sang qui sort de ces organes est chargé de déchets azotés, il doit donc passer le plus vite possible par les reins pour être nettoyé ou filtré. Avant enseignement, les élèves s'inscrivent donc dans un cadre de nutrition directe avec un acheminement sanguin sans détours de substances nutritives du milieu extérieur vers les organes et une évacuation immédiate des déchets. La logique d'un système avec des organes en série fait que le muscle reçoit au plus vite ce dont il a besoin et élimine ses déchets sans trop salir le sang. Nous retrouvons dans cette construction d'un modèle en série, un raisonnement par mise en histoire qui facilite leur compréhension du problème en le simplifiant.

Le modèle en parallèle ne peut être compris que par une approche systémique qui ne sépare pas dans le temps les différents événements: à un instant donné, le compartiment sanguin contient une certaine quantité d'urée bien en dessous de la concentration limite; à tout moment, les organes provoquent des entrées d'urée dans ce compartiment et les reins assurent des sorties. Si les sorties sont égales aux entrées, l'état normal se maintient. Il ne s'agit donc pas d'une logique d'évacuation mais d'équilibre dynamique dans laquelle les élèves peinent à entrer. D'ailleurs l'analyse dans un deuxième temps du schéma de la figure $4 \mathrm{~b}$ les met très généralement dans l'embarras. Ils conçoivent mal la place du tube digestif si loin, dans le circuit, des organes qu'il doit approvisionner, et surtout celle des reins qui fait que le sang est partout sale.

Pour résumer, les lycéens construisent le problème d'approvisionnement des organes en substances nutritives et l'évacuation de leurs déchets dans une approche de 
nutrition directe d'un organisme animal et avec la mise en jeu d'un milieu intérieur conçu comme un vecteur de substances dont on narre le parcours entre le milieu extérieur et les organes (mise en histoire simple ou storytelling). C'est donc que les deux bascules bernardiennes (voir la section 3 de cet article) ne sont pas faites par les élèves, ce qui fait douter qu'ils aient une approche unitaire des animaux et des végétaux.

\section{Le problème du renouvellement moléculaire des cellules}

Le travail du problème du renouvellement moléculaire au sein des cellules de l'organisme relève d'une approche systémique du fonctionnement du milieu intérieur. Le deuxième cas que nous étudions met en jeu deux lycéennes de première scientifique (grade 11, 16-17 ans) en train de s'entretenir après le cours sur ce sujet avec leur professeur de sciences de la vie et de la Terre. C'est une autre façon de cerner le rapport des élèves à la physiologie et au principe d'unité des animaux et des végétaux qui la structure.

\section{Le contexte de l'entretien}

60 Nous partons d'un cas où tous les élèves d'une classe de première scientifique ont réfléchi individuellement puis en binômes au fonctionnement d'une cellule animale. La confrontation des travaux a permis de construire un schéma donnant une vue globale $\mathrm{du}$ fonctionnement de l'organisme et d'une cellule quelconque de cet organisme. Si le contenu de ce schéma résume les processus (respiration et synthèses cellulaires) auxquels ont fait référence les élèves, le type de schématisation choisi par le professeur est celui illustré par la figure 5.Le but est de se dégager de la représentation classique " circulation sanguine/ cellules ou organes » qui favorise un raisonnement séquentiel pour une modélisation de type systémique (voir la section précédente).

Figure 5. Schéma du fonctionnement global d'un organisme humain

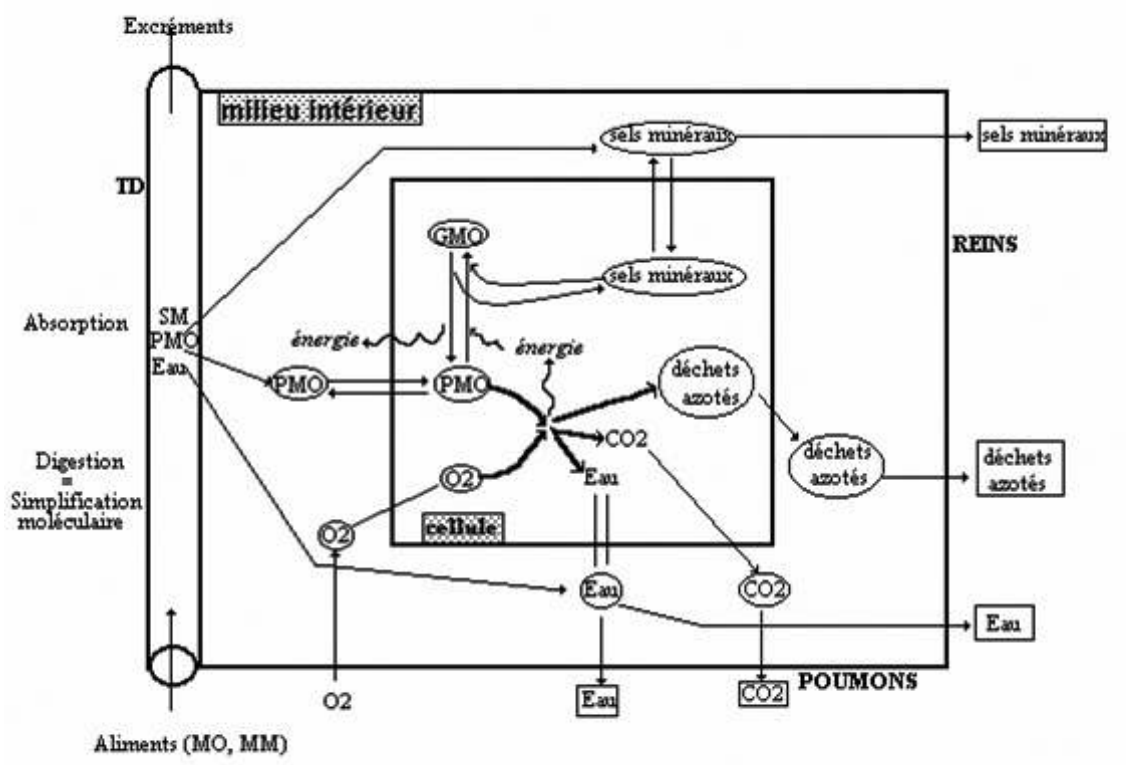


61 Ce schéma n'est pas encore terminé : il a été convenu avec les élèves de poursuivre sa construction au fur et à mesure. C'est ainsi que l'étude du renouvellement moléculaire a été entreprise. Le professeur a alors demandé à la classe d'expliquer l'évolution de la radioactivité des protéines hépatiques de rats contenant des acides aminés radioactifs. Les élèves disposent pour cela d'une courbe montrant cette évolution au cours du temps. Cette étude doit aboutir à la notion de renouvellement moléculaire et permettre de compléter le schéma de fonctionnement cellulaire : ajout, dans la cellule du schéma, d'une flèche allant des grosses Molécules Organiques (gMO) aux petites Molécules Organiques (pMO) car les grosses molécules organiques redonnent des petites molécules organiques.

62 C'est après ce travail que deux élèves (Sophie et Jessica) ont sollicité un entretien auprès du professeur, demande justifiée par les difficultés qu'elles éprouvent à faire fonctionner seules le grand schéma. Avec leur accord, cet entretien est enregistré. Nous nous proposons d'y rechercher les conceptions qu'elles ont du milieu intérieur.

\section{Les marques d'une mise en histoire simple dans l'entretien}

Jessica et Sophie n'arrivent plus, seules, à bien faire fonctionner le modèle de cellule travaillé dans le cours. Elles ont pourtant demandé l'aide et l'avis de plusieurs de leurs camarades. Par quoi se caractérise leur raisonnement? Nous relevons que de manière récurrente Sophie, qui s'exprime davantage, séquentialise les processus. Prenons l'intervention 31 :

$31 \mathrm{~S}$ : Donc en fait, de toute façon, c'est la respiration cellulaire qui se fait en premier ${ }^{11}$ parce que pour faire des gMO, y a besoin d'énergie. Donc quelque part, y a besoin qu'au départ, il y ait eu de la respiration qui se soit faite.

Ainsi, dans un premier temps, la respiration se déroule et produit de l'énergie. Puis, dans un deuxième temps, il y a synthèse de grosses molécules organiques (gMO) grâce à cette énergie.

Dans la suite de la discussion, où au contraire du professeur, les deux élèves s'impliquent fortement, elles établissent que les petites molécules organiques (pMO) qui servent dans la respiration sont définitivement perdues et que certaines pMO peuvent être enrôlées plusieurs fois dans le cycle construction/démolition de grosses molécules organiques (gMO) ou entrer définitivement dans la respiration. Ce faisant, elles élaborent des possibles de devenirs moléculaires. L'extrait suivant le montre :

$51 \mathrm{~S}$ : Ben oui, mais c'est les mêmes! Puisque je veux dire que quand tu fais des pMo, quand tu casses, quand elles se cassent... Enfin, quand elles se rassemblent ça fait des gMO et quand elles se cassent ça fait les mêmes pMO... C'est un cycle sans fin en fait c'est toujours les mêmes pMO qui serviraient, je sais pas, je trouve...

$6852 \mathrm{~J}$ : Mais non, parce que les pMO qui servent à la respiration, y en a plus après! Donc les pMO qui servent avec l'énergie pour moi c'est celles qui vont...

Notons que leurs propos montrent qu'elles n'écartent pas totalement la mise en séquence de l'explication :

$53 \mathrm{~S}$ : Donc, en fait, c'est bien ça qui se produirait en premier la respiration.

$54 \mathrm{~J}:$ Oui, euh...

$55 \mathrm{~S}:$ Donc à l'origine tu manges des pMO, les pMO s'en vont directement avec l'O2 elles font l'énergie et t'as d'autres pMO qui arrivent pour... 
Lorsquent dintegration est abordé au lycée et dans lenseignement supérieur. Lorsqu'on propose à des professeurs des écoles stagiaires d'expliquer le mouvement du membre supérieur suite à une stimulation d'un de ses doigts, le plus classiquement et le plus fréquemment, on trouve ce fameux centralisme cérébral, c'est-à-dire que les afférences sensorielles vont directement au cerveau, le cerveau traite l'information et envoie l'information correspondante : excitation du muscle fléchisseur et inhibition du muscle extenseur. Il n'y a pas du tout d'intervention des réseaux de neurones qui interviennent au niveau de la moelle épinière pour assurer le fonctionnement coordonné des muscles antagonistes que sont le biceps et le triceps. Spontanément, les élèves et les étudiants raisonnent sans envisager une possibilité de fonctionnement du centre nerveux médullaire. C'est une marque de non mise en jeu du principe d'unité de fonctionnement des cellules nerveuses. 
Pourtant, dans les débats scientifiques autour de ce type de productions, certaines propositions peuvent être interprétées comme porteuses du principe d'unité de fonctionnement tels que les extraits ci-dessous :

rina : Oui je réalise que le nerf optique c'est un nerf; donc à partir de l'œil il y a déjà un nerf donc il va se passer quelque chose d'identique à ce qui peut se passer dans le cerveau et en réponse puisque c'est un nerf.

87 164. Sabrina: Je me dis que les nerfs ça fonctionne de la même façon forcément ${ }^{12}$, ce qui veut dire que dès le nerf optique, ce qui va se passer là ça va à peu près ça va être le même style.

166. Sabrina : Ça va être la même chose qui va se passer quand l'information va être donnée par le cerveau, donnée aux nerfs.

Figure 6. Exemple de production d'étudiants préparant le concours de professeur des écoles

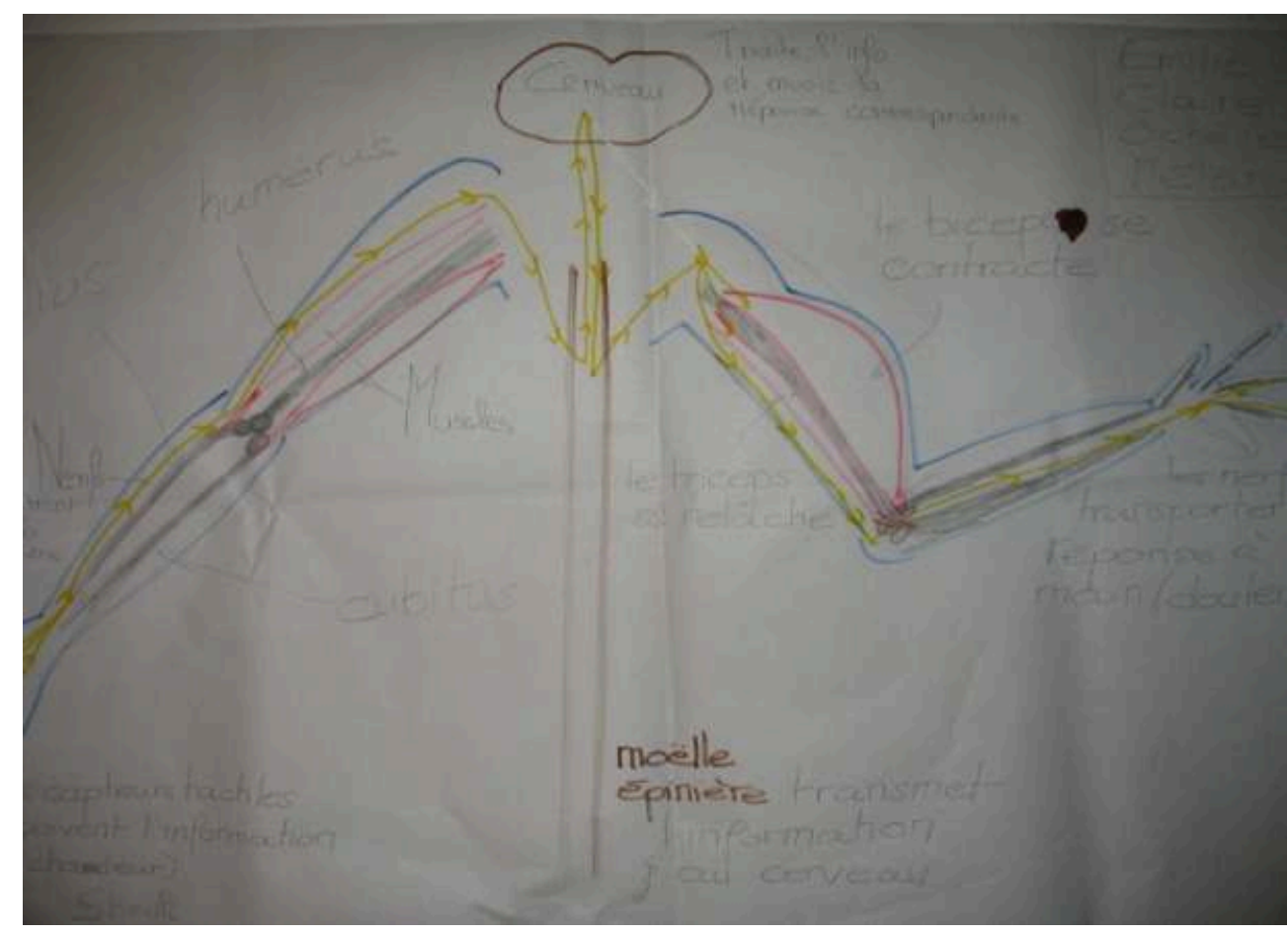

Le principe d'explication mobilisé dans les propos de Sabrina peut se résumer de la façon suivante: quel que soit l'endroit dans le système nerveux, les structures nerveuses fonctionnent de la même façon. Cela semble se rapprocher du principe sur lequel s'est appuyé Sherrington lors de l'élaboration du concept d'intégration. Cependant, il apparait comme peu élaboré puisqu'il n'est pas mis en relation avec la nécessité d'intégration et ne concerne le fonctionnement que d'un point de vue global. D'autre part, il fonctionne comme un postulat sans référence à une quelconque contrainte empirique. Chez Sherrington, le principe mobilisé d'une part se fonde sur des théories (cellulaire et de l'évolution) et d'autre part structure la problématique de recherche qui articule construction des concepts et faits d'observation ou expérimentaux. Cela n'est pas du tout le cas, dans les propos cités ci-dessus. Ce principe de premier niveau mis en jeu par l'étudiante sert seulement à expliquer une transmission nerveuse selon des modalités communes à l'ensemble du système nerveux mais n'a pas de fonction dans l'élaboration d'une explication qui concernerait 
l'intégration soit au niveau de la moelle épinière soit au niveau du cerveau, qui implique des structures telles que les neurones et les synapses.

\section{Conclusion}

La réflexion que nous venons de conduire montre que les principes auxquels nous avons prêté attention (principe de l'actualisme, principe d'unité de fonctionnement des animaux et des végétaux, principe d'unité de fonctionnement des cellules nerveuses) tirent leur heuristicité du fait qu'ils contribuent d'une part à extraire de la pensée immédiate pour accéder à une pensée scientifique, d'autre part à faire vivre le monde des problèmes scientifiques (le $3^{\mathrm{e}}$ monde de Popper) parce qu'ils permettent de dépasser certaines tensions constitutives des disciplines scientifiques (tensions entre diversité et unité, entre stabilité structurelle et changements permanents, entre fonctionnement à un moment donné et histoire, entre déterminisme et autonomie, entre continuité et discontinuité, etc.). Ils sont fondateurs de champs scientifiques et dit autrement, ils participent de la gestion des apories fondatrices des sciences (Thom, 1983). Dans cette dernière partie, nous voulons récapituler quelques-uns de leurs rôles.

\section{Un barrage contre les irrationalismes}

91 Au terme de cette étude, nous aboutissons à l'idée que la fécondité des principes que nous avons étudiés tient notamment au fait qu'ils s'érigent contre des irrationalismes. Nous prendrons ces irrationalismes de deux manières :

- sous l'angle de constructions explicatives liant sans discernement ordinaire et extraordinaire et fortement empreintes de catastrophisme de bas niveau ;

- sous l'angle d'un type de raisonnement que nous avons qualifié de mise en histoire ou storytelling, conduisant à des formes de récits peu contrôlés et fermant les problèmes.

Comme l'écrit Bruner, les récits domestiquent l'inattendu et l'exceptionnel, assemblent le disparate et le fragmentaire, ce qui leur permet de donner forme et sens au monde qui nous entoure : «La fonction de l'histoire est de trouver une intention qui atténue ou au moins rende compréhensible une déviation par rapport à un élément culturel canonique. » (1997, p. 63). Si dans la pensée commune, ils concernent surtout l'ici et maintenant, ils ne s'y limitent pas. Ils aident en effet à construire et à inventer le passé et l'avenir, dans un processus où mémoire et imagination se mêlent, se nourrissent et s'entretiennent (Bruner, 2002, p. 82).

Certes ces caractéristiques valent également pour les sciences qui, selon Popper, imaginent des histoires pour expliquer les phénomènes naturels : elles sont en cela proches des mythes mais s'en distinguent par une tradition d'analyse critique des récits produits. Les mythes scientifiques ne sont pas, écrit-il, «des sublimés de nos observations » (Popper, 1985, p. 194). Ils sont toujours au départ des fictions. Mais, sous la pression de la critique, « ils sont contraints de s'adapter à la finalité de nous donner une représentation adéquate et plus détaillée du monde où nous vivons » (ibid., p. 194).

Pour les domaines scientifiques ayant une composante historique marquée (la géologie, la biologie évolutionniste), où il s'agit de reconstruire des phénomènes et des événements en des temps où l'homme n'existait pas, les principes structurants comme celui de l'actualisme méthodologique font figure de garde-fou tenant à l'écart de 
certaines formes de fictions. On peut questionner plus encore leur portée et se demander si d'autres principes théoriques forts guident aussi l'activité scientifique dans ses relations avec l'histoire, le temps et... les récits. Nous pensons notamment à la construction des problèmes de développement des êtres vivants où la vie et la mort cellulaires se conjuguent (Ameisen, 2003).

\section{Des changements de niveau d'explication}

En reprenant quelques-uns de nos exemples (physiologie nerveuse, géologie), il est possible de montrer que la mise en jeu de ces principes peut accompagner des changements de niveau d'explication au sein d'un même paradigme, voire même des changements de paradigme.

96 - Le principe d'unité de fonctionnement des cellules nerveuses permet de penser des fonctionnements nerveux en dehors de la zone cérébrale. Il se pose donc contre un centralisme cérébral tout en maintenant la possibilité de le retrouver, mais dans un rapport de nécessité. Ce principe élimine une mise en jeu immédiate du cerveau, et les représentations du sens commun qu'elle véhicule, pour mieux le retrouver. De plus, sa tenue a une portée heuristique puisque, et l'histoire des sciences en témoigne, il permet de construire de nombreux concepts.

97 - Le principe de l'actualisme, en exportant dans le passé des causes encore agissantes tend à fonctionner contre l'histoire pour mieux la retrouver. De fait, il permet de construire la nécessité du temps long constructeur de phénomènes, celle de causes passées différant en nature ou en intensité des causes actuelles, et même de construire des événements historiques singuliers dans le temps et dans l'espace. C'est lorsque le principe méthodologique de l'actualisme a été porté à ses limites que les géologues du $\mathrm{XIX}^{\mathrm{e}}$ siècle ont été contraints et forcés d'envisager une forme élaborée de catastrophisme.

\section{L'accès à la maîtrise du fonctionnement des systèmes complexes}

Ce point est lié au précédent. Les systèmes biologiques et géologiques tiennent leur complexité du fait que leur fonctionnement et leur histoire convoquent une diversité de processus se déployant dans des dimensions spatiotemporelles variées, certains jouant localement quand d'autres concernent le système entier, certains étant synchrones quand d'autres se succèdent. Comment par exemple tenir le "en même temps » quand le « et puis » tend à le remplacer? Nous savons en effet que la mise en histoire tend à tout linéariser. C'est aussi ce que font certaines approches causales (Viennot, 2003). Cela contrarie la construction des problèmes où il s'agit de rendre compte d'une stabilité structurelle dynamique (problème de renouvellement moléculaire ou de renouvellement des fonds océaniques, etc.) et d'adopter un raisonnement systémique. Certains principes structurants y contribuent plus par les concepts qu'ils permettent de construire que dans une application directe (voir le principe d'unité et de diversité de fonctionnement des animaux et des végétaux et le concept de milieu intérieur). L'étude du fonctionnement d'un système biologique ou géologique oblige donc à penser la mobilisation de ces principes mais aussi des situations où les élèves prennent en charge conjointement des problèmes ne se déployant pas dans une même échelle spatiale et où le temps des phénomènes marque 
différemment l'espace (Orange Ravachol, 2007). Les principes structurants sont donc plus généraux que les nécessités portant sur les modèles explicatifs, qui elles sont spécifiques d'un problème donné. Mais ils pilotent fortement la construction de l'espace des contraintes caractéristiques du travail de chacun de ces problèmes et donc la construction de ces nécessités. Il reste à préciser plus finement l'étendue des champs disciplinaires ou des champs de problèmes pour laquelle la fonction structurante de ces principes est tenue pour vraie. Nous nous y employons en étudiant la place du vrai et du faux dans la problématisation en sciences de la vie et de la Terre.

\section{BIBLIOGRAPHIE}

Albritton, C.C. (1967). Uniformity, the Ambiguous Principle. In Uniformity and Simplicity, A Symposium on the Principle of the Uniformity of Nature. Boulder, Colorado: The Geological Society of America, Incorporated, 1967. p. 1-2.

Ameisen, J.-C. (2003). La Sculpture du vivant. Paris : Le Seuil, Points sciences.

Babin, C. (2005). Autour du catastrophisme. Paris : Vuibert-ADAPT.

Bachelard, G. (1972). L'Engagement rationaliste. Paris : PUF.

Bachelard, G. (1986/1949). Le Rationalisme appliqué. Paris : Quadrige/PUF.

Bachelard, G. (1938). La Formation de l'esprit scientifique. Paris : Vrin.

Beorchia, F. (2003). La Communication nerveuse : conceptions des apprenants et problématisation. Importance des explications mécanistes et vitalistes. Thèse de doctorat, Université de Nantes.

Bernard, C. (1966/1865). Introduction à la médecine expérimentale. Paris : Garnier Flammarion.

Bernard, C. (1877). Leçons sur le diabète et la glycogenèse animale. Paris : J.-B. Baillière et fils.

Bruner, J. (2002). Pourquoi nous racontons-nous des histoires ? Paris : Retz.

Bruner, J. (1997/1991). ... Car la culture donne forme à l'esprit. Paris : Eshel (1991).

Canguilhem, G. (1988). Idéologie et rationalité dans l'histoire des sciences de la vie. Paris : Vrin.

Canguilhem, G. (1983). Études d'histoire et de philosophie des sciences. Paris : Vrin.

Canguilhem, G. (1955). La Formation du concept de réflexe aux XVII et XVIII siècles. Paris : PUF.

Cêlal Sengör, A.M. (2005). Une autre histoire de la tectonique. Paris : Collège de France/ Fayard.

Celâl Sengör, A.M. (2001). Is the Present the Key to the Past or the Past the Key to the Present? Boulder : The Geological Society of America, special paper 355.

Ellenberger, F. (1996). Le présent, clef du passé. In Travaux du comité français d'histoire de la géologie (COFRHIGEO), troisième série, t. X, nº 5 (séance du 20 mars 1996).

Ellenberger, F. (1994). Histoire de la géologie, tome 2. Paris : Technique et Documentation (Lavoisier). 
Fabre M. \& Orange C. (1997). Construction des problèmes et franchissement d'obstacles. ASTER, 24, 37-57 (disponible sur http://documents.irevues.inist.fr/handle/2042/8550, consulté le 10 janvier 2011).

Gohau, G. (2003). Naissance de la géologie historique. Paris : Vuibert.

Gohau, G. (1997). Naissance de la méthode « actualiste » en géologie. In Gohau G. dir., (1997). De la géologie à son histoire, CTHS, p. 139-149.

Gould, S.J. (1990). Aux racines du temps. Paris : Grasset. (1987).

Gould, S.J. (1989). Wonderful Life, The Burgess Shale and the Nature of History. New York : W.W. Norton \& Company, Inc. (1991, La vie est belle, Paris : Le Seuil, pour l'édition française).

Gould, S.J. (1965b). Is uniformitarianism necessary ? ; a reply. American Journal of Science, vol. 263, December 1965, p. 919-921.

Gould, S.J. (1965a). Is uniformitarianism necessary? American Journal of Science, vol. 263, March 1965, p. 223-228.

Grmek, M.D. (1997). Le Legs de Claude Bernard. Paris : Fayard.

Hooykaas, R. (1963). The Principle of Uniformity in Geology, Biology and Theology. Leiden : E.J. Brill. (1970). Continuité et discontinuité en géologie et biologie. Paris : Le Seuil, pour l'édition française).

Jacob, F. (1981). Le Jeu des possibles. Paris : Fayard.

Lévi-Strauss, C. (1962). La Pensée sauvage. Paris : Plon.

Mayr, E. (1989). Histoire de la biologie. Paris : Fayard.

Orange, C. (1997). Problèmes et modélisation en biologie. Paris : PUF.

Orange, C., coord., Orange, D., Le Bot, F. \& Michaud, M. (2000). Changer, réorganiser, refondre ses connaissances en biologie. Rapport de la recherche INRP/IUFM des Pays de la Loire 1997/2000.

Orange Ravachol, D. (2007). Des mises en histoire aux savoirs scientifiques : le cas de lycéens confrontés à quelques problèmes de tectonique des plaques. ASTER, 44, 41-68.

Orange Ravachol, D. (2005). Le principe de l'actualisme, pierre d'achoppement des lycéens confrontés à des problèmes de Géologie historique. Actes des $4^{\text {es }}$ rencontres scientifiques de l'ARDIST, Lyon, octobre 2005, p. 265-272. (disponible sur : http://ardist. aix-mrs.iufm.fr/, consulté le 10 janvier 2011).

Orange, D. (2003b). Tendance à la « mise en histoire " par les élèves de lycée en sciences de la vie et de la Terre : étude de deux cas. Actes des $3^{\text {es }}$ rencontres scientifiques de l'ARDIST, Toulouse, octobre 2003, p. 239-246. (disponible sur : http://ardist.aix-mrs.iufm.fr/, consulté le 10 janvier 2011).

Orange Ravachol, D. (2003a). Utilisations du temps et explications en sciences de la Terre par les élèves de lycée : étude dans quelques problèmes géologiques. Thèse de doctorat. Université de Nantes, (disponible en ligne sur http://tel-archives-ouvertes.fr/, consulté le 10 janvier 2011).

Orange Ravachol, D. \& Beorchia, F. (2007). Principes structurants et savoirs en sciences de la vie et de la Terre. Actes du Congrès de l'AREF, Strasbourg, 29 août- $1^{\mathrm{er}}$ septembre 2007, (disponible sur : http://www.congresintaref.org/actes_site.php, consulté le 10 janvier 2011).

Orange, C. \& Orange, D. (1995). Biologie et géologie, analyse de quelques liens épistémologiques et didactiques. ASTER, 21, p. 27-49, (disponible sur http://documents.irevues.inist.fr/handle/ 2042/8540, consulté le 10 janvier 2011). 
Oreskes, N. (2001). Plate Tectonics, An Insider's History of the Modern Theory of the Earth. Boulder, Oxford: Westview Press.

Oreskes, N. (1999). The Rejection of Continental Drift. New York, Oxford : Oxford University Press.

Popper K. (1979). Objective knowledge. Oxford University Press. (1991, Paris : Aubier, pour l'édition française)

Popper K. (1972). Conjectures and refutations. London : Routledge and Kegan Paul (1985, Conjectures et réfutations, Paris : Payot, pour l'édition française).

Rudwick, M.J.S. (2008). Worlds before Adam. Chicago : The University of Chicago Press.

Rudwick, M.J.S. (1962). «The principle of Uniformity », a review of R. Hooykaas, Natural Law and Divine Miracle (Brill, Leiden, 1959). In History of Science, 1962, I, 82-86.

Shea, J.H. (1982). Twelve fallacies of uniformitarianism. In Geology, september 1982, v.10, p. 455-460.

Sherrington, C. S. (1906). The integrative action of the nervous system. New-York : Charles Scribner's Sons.

Thom (1983). Paraboles et catastrophes. Paris : Flammarion.

Viennot, L. \& Debru, C. (2003). Enquête sur le concept de causalité. Paris : PUF.

\section{NOTES}

1. L'adjectif actuel de l'expression «principe des causes actuelles» résulte d'une mauvaise traduction de l'anglais actual. Il signifie réel et non actuel.

2. «Actuelle » est ici pris dans sa signification française.

3. Voir par exemple les cosmogonies de Burnet, Woodward et Whiston auxquelles renvoient Hooykaas (1970, p. 18) et Gould (1990, p. 42).

4. Une terminologie que l'on doit au géologue et logicien anglais $\mathrm{W}$. Whewell (1794-1866).

5. Cette conception d'une Terre qui se contracte, admise à l'époque, a depuis été réfutée.

6. Hooykaas range Elie de Beaumont dans les catastrophistes actualistes (1970, p. 22).

7. Auxquels nous pourrions ajouter notamment les travaux de Albritton (1967), Celâl Sengor (2001), Rudwick (1962, 2008), Shea (1982).

8. Cette appréciation est désormais contestable du fait de la récente prise d'importance de courants fondamentalistes.

9. Le problème de la formation d'une chaîne de montagnes intracontinentale est abordé dans la classe de $4^{\mathrm{e}}$ du collège (grade 8 , élèves de 13/14 ans) et dans la classe de terminale scientifique du lycée (grade 12, élèves de 17-18 ans).

10. Ces deux cas se rapportent à l'ancien programme de première S (1992).

11. Surligné par nous.

12. Surligné par nous. 


\section{RÉSUMÉS}

Dans cet article, nous travaillons sur les principes structurants, qui organisent les domaines de savoirs scientifiques mais dont l'affichage est limité dans les programmes d'enseignement actuel. Nous recourons à trois exemples dont l'importance est reconnue dans le fonctionnement des sciences de la vie et de la Terre: le principe de l'actualisme, le principe d'unité de fonctionnement des animaux et des végétaux, le principe d'unité de fonctionnement des cellules nerveuses. Notre étude, qui s'inscrit dans le cadre théorique de la problématisation, construit des repères épistémologiques permettant de caractériser l'appropriation par les élèves et les étudiants des problèmes de la géologie historique et de la physiologie, et de préciser les rapports qu'entretiennent leur raisonnement avec ces principes structurants. Elle met en valeur la portée heuristique de ces principes, leur intervention dans l'accès à la maîtrise du fonctionnement de systèmes complexes, et plus généralement leur rôle de barrage contre les irrationalismes.

Abstract: In this article, we work on the structuring principles that organize the fields of scientific knowledge whose display is limited in current teaching programs. We use three examples whose importance is recognized in the operation of life and Earth sciences: the principle of uniformitarianism, the principle of unity of functioning of animals and plants, the principle of unity of functioning of the nerve cells. Our study, which takes place within the theoretical framework of the problematization, allows to characterize the epistemological appropriation by pupils and students of the historical geology and physiology problems, and to clarify the relationship of their reasoning with these structuring principles. It highlights the scope of these heuristic principles, their intervention in access to the control of operation of complex systems, and more generally their role of barrier against irrationalism.

\section{INDEX}

Keywords : scientific knowledge, problematization, structuring principles, learning, life sciences and Earth science

Mots-clés : savoirs scientifiques, problématisation, principes structurants, sciences de la vie et de la Terre, apprentissage

\section{AUTEURS}

\section{DENISE ORANGE RAVACHOL}

(université de Nantes-IUFM Pays de la Loire, CREN)

\section{FRANÇOISE BEORCHIA}

(université de Caen-IUFM Basse-Normandie, CERSE) 